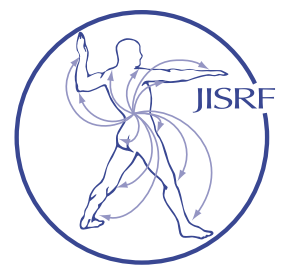

\title{
Low Transfusion Rate Attainable in Anterior Approach Total Hip Arthroplasty Utilizing a Modern Protocol
}

\author{
Gondusky, $\mathrm{J}^{1}$; Campbell, $\mathrm{B}^{2}$; Coulson, $\mathrm{C}^{3}$
}

\begin{abstract}
Abstact
Background: Transfusion is a known risk of total hip arthroplasty (THA). It has been associated with a multitude of medical complications and increased cost. Prior studies report transfusion rates associated with THA, with wide variation, but most cannot differentiate the surgical approach utilized. The anterior approach (AA) for THA has been associated with increased operative time, complications, and blood loss, but little data exists regarding the actual transfusion rate associated with the approach.
\end{abstract}

Methods: We performed a retrospective review of 390 consecutive, elective, primary unilateral AA THA procedures. Patient demographic, clinical and perioperative data was analyzed. A modern perioperative pathway, including a simple protocol to limit blood loss, is defined.

Results: The group consisted of a typical inpatient arthroplasty population, with wide ranges of age, body mass index (BMI), and health status. The average age was 64.05 years $(+10.67$, range $27-94)$. BMI averaged $29.76 \mathrm{~kg} / \mathrm{m} 2$ (+5.98, range 16-47). The majority of patients were American Society of Anesthesiologists (ASA) class 2 (45.6\%) or $3(50.3 \%)$, with 10 patients ASA 4 (2.6\%). Average preoperative hemoglobin was $13.48 \mathrm{~g} / \mathrm{dL}(+1.47$, range 9.118.2). Operative time averaged 91.22 minutes $(+14.2)$. $83.3 \%$ of patients received a spinal anesthetic. Most patients were discharged on postoperative day one (93.1\%) to home (99\%). Estimated blood loss averaged $264 \mathrm{~mL}$ (+ 95.19, range 100-1000). No patient required perioperative transfusion or readmission for symptomatic anemia within
30 days postoperative.

Conclusion: A modern protocol we utilize and define is capable of limiting blood loss and transfusion risk in anterior approach total hip arthroplasty.

\section{Background}

Substantial blood loss and the resultant need for perioperative transfusion is a known risk of most major surgical procedures, including total hip arthroplasty (THA). Transfusion is associated with risks to the patient, and significant cost to the healthcare system [1- $\underline{6}]$. Mitigating the risk of this complication should be one of the goals of successful primary THA.

Wide variation exists in the reported rate of transfusion in primary, elective THA. Modern studies report transfusion rates between $0-39.2 \%$ [1- $\underline{4}, \underline{6}-\underline{20}]$. In large scale studies of the topic, wide variation is noted amongst hospitals. This is attributed to differences in procedural volume and length of stay $[\underline{6}, \underline{15}]$. The most recent, large patient volume analyses, demonstrate a transfusion rate range from $11.8-22.2 \%[\underline{4}, \underline{6}, \underline{10}, \underline{12}, \underline{15}]$. Notably, some of these studies highlight a concerning trend toward an increased rate of transfusion over time $[\underline{4}, \underline{6}]$.

Transfusion is not without risk. Hemolytic and allergic

Keywords: anterior approach; direct anterior; primary total hip arthroplasty; total hip arthroplasty; transfusion; blood loss Level of Evidence: IV, Retrospective Case Series 
reactions, circulatory overload, acute lung injury, and disease transmission have been reported [1- $\underline{3}]$. In addition, transfusion has been associated with immunomodulatory effects and increased risk of perioperative infection, including surgical site and respiratory infection. In joint arthroplasty, transfusion has been associated with increased hospital length of stay (LOS), likelihood of discharge to a skilled nursing facility (SNF), mortality, and cost $[\underline{1}, \underline{2}, \underline{4}-\underline{6}]$. Estimates of hospital cost associated with a single transfusion in knee arthroplasty patients was found to be $\$ 1777$ [20]. In an era where quality outcomes have been linked to payment via bundled care arrangements, and there is increased movement of THA to outpatient centers without transfusion capability, it is important to avoid this medical, logistical and financial risk.

The anterior approach (AA) for THA has been associated with increased operative time, blood loss, and complications, especially during the procedural "learning curve." [21-30]. A continued increase in operative time and blood loss with AA THA, relative to the posterolateral approach, has been suggested even beyond the learning curve [31]. The blood loss reported in series outside of the learning curve for AA THA averages $320-463 \mathrm{~mL}$, with a range of $50-2000 \mathrm{~mL}[\underline{28}, \underline{31}-\underline{34}]$. Learning curve studies reveal a reported maximum estimated blood loss (EBL) range up to $2718 \mathrm{~mL}$ [22]. While self-reported EBL can be an unreliable and subjective metric, transfusion rate is objective and has been shown to be associated with increased perioperative risk and cost, but it too can be confounded by autologous pre-donation, patient selection bias, or the use of variable transfusion triggers $[\underline{1}-\underline{6}, \underline{20}]$. Transfusion data is rarely reported with information regarding the approach utilized for elective THA. No large-scale study exists which reports transfusion data for AA THA. Smaller studies available are confounded by autologous transfusion $[\underline{24}, \underline{35}]$ or selection bias [34]. Given data that suggests blood loss is higher with the AA, it could be suspected that the AA THA transfusion rate may be higher than that reported regardless of approach, but little objective and unbiased data is available.

Determining the rate and preventing the occurrence of transfusion in AA THA is important to improve patient care and quality outcomes, move AA THA toward the outpatient setting, and minimize cost. The goal of this study was to determine the rate of transfusion in a consecutive series of AA THA patients typical of an inpatient setting. We also attempt to define a simple, modern protocol to reduce perioperative blood loss and transfusion risk about AA THA.

\section{Materials and Methods}

Once institutional review board approval was obtained, we queried the hospital's electronic medical records to identify consecutive patients who underwent primary, unilateral, elective THA via the anterior approach (AA) by a single surgeon between January 2018 and November 2019. A total of 390 cases were identified. No patients were excluded. Conversion and revision cases were not included.

The surgeon is a high volume, fellowship trained arthroplasty surgeon beyond the learning curve, with no strict contraindications to AA THA (no primary THA via an alternative surgical approach was performed during the study period). All surgeries were performed on a table allowing leg manipulation (hana, Mizuho OSI, Union City, California). The surgical technique utilized is as per that described previously [ㅎ6].

All patients underwent standard preoperative lab testing, including hemoglobin/hematocrit $(\mathrm{Hg} / \mathrm{HCT})$ and coagulation levels. No blood pre-donation, or preoperative iron or other supplemental medical treatment was utilized. There was no absolute exclusion criteria based on preoperative anemia. Standard discontinuation of preoperative anticoagulants was done according to specialist consultant, primary care, or anesthesia recommendations. Preoperative primary care clearance was obtained. Specialist clearance was obtained as needed, most commonly for significant cardiac history.

Intraoperatively, no blood collection or autotransfusion device was utilized. Hemostasis was obtained with standard bovie electrocautery, with meticulous hemostasis the goal at each surgical plane. The specific surgical steps deemed most important to minimizing blood loss are defined within Table 1 and the Discussion section.

Use of spinal anesthesia was preferred and planned. All patients received intravenous (IV) tranexamic acid (TXA), one gram at the initiation of the case and one gram IV during final closure. Intravenous dexamethasone (10mg) was also given at the start of the procedure to aid in control of pain and nausea. This was withheld in insulin dependent diabetic patients or those with high preoperative blood sugar if felt optimal, in coordination with the anesthesia team. No specific hypotensive anesthesia protocol was employed. Generally, hypotensive anesthesia was attempted and preferred, but logistically this varied by anesthesia provider and patient. At the conclusion of the case, a total of $90 \mathrm{~mL}$ mixture of ropivacaine $(60 \mathrm{~mL}, 0.5 \%)$ and ketorolac $(1 \mathrm{cc}, 30 \mathrm{mG} / \mathrm{mL})$, diluted in normal saline, was instilled into the hip capsule and subcutaneous tissues. Standard perioperative antibiotics were given. Postoperative DVT prophylaxis was predominantly aspirin (ASA), 
81 or $325 \mathrm{mg}$ twice a day for four weeks, started within hours postoperatively. No drains were utilized. Closure proceeded with capsule, fascia, subcutaneous and skin. Skin closure was with absorbable subcuticular suture and a skin glue strip, followed by application of a water impermeable dressing. The perioperative aspects felt most important to limit excessive blood loss and transfusion are summarized in Table 1.

Table 1. Protocol Steps to Minimize Blood Loss and Transfusion Rate in Anterior Approach THA

\begin{tabular}{|c|c|}
\hline Preoperative & $\begin{array}{l}\text { - Optimize preoperative hemoglobin } \\
\text { - Ensure preoperative coagulation labs normal, } \\
\text { check LFTs if indicated } \\
\text { - Discontinue NSAIDs and anticoagulant } \\
\text { medicines according to guidelines and specialist } \\
\text { recommendations }\end{array}$ \\
\hline Intraoperative & $\begin{array}{l}\text { - Intravenous and/or topical TXA use } \\
\text { - Neuraxial anesthesia favored over general } \\
\text { endotracheal } \\
\text { - Standard bovie electrocautery } \\
\text { - Meticulous surgical hemostasis* } \\
\text { - Bone wax use on the cut femoral neck, as } \\
\text { needed } \\
\text { - Blood pressure management } \\
\text { - Minimize operative time } \\
\text { - Avoid drain use } \\
\text { - Adequate preoperative and intraoperative } \\
\text { hydration }\end{array}$ \\
\hline Postoperative & $\begin{array}{l}\text { - Choose an optimal anticoagulant agent } \\
\text { - Nursing-based fluid resuscitation protocol for } \\
\text { hypotension } \\
\text { - Utilize evidence-based transfusion triggers [글 }\end{array}$ \\
\hline
\end{tabular}

LFTs, liver function tests; NSAIDS, nonsteroidal anti-inflammatory drugs; TXA, tranexamic acid

*see notes in the article text regarding sequential surgical steps recommended to achieve optimal hemostasis

Postoperatively, all patients received internal medicine hospitalist consultation for management of medical issues. These providers and the surgical team assessed the patient clinically for concern of symptomatic hypotension. Routine postoperative labs were not ordered. Parameters for the treatment of hypotension were standardized and allow for the nursing staff to administer $500 \mathrm{~mL}$ fluid boluses at blood pressure thresholds. Our transfusion triggers are as recommended by established clinical practice guidelines, with consideration of transfusion for $\mathrm{Hg}$ of $8 \mathrm{~g} / \mathrm{dL}$ or less, or for symptoms of chest pain, orthostatic hypotension, tachycardia unresponsive to fluid resuscitation, and in the setting of congestive heart failure [37]. Any of these scenarios were discussed in conjunction with our hospitalist colleagues to determine if transfusion was deemed in the best interest of the patient.

A standard multimodal pain control pathway was fol- lowed, utilizing local ice, oral acetaminophen, IV nonsteroidal anti-inflammatory (NSAID), IV dexamethasone (10mg AM POD \#1), and limited narcotics. NSAIDs were withheld in patients with chronic kidney disease (CKD), or based on the judgement of the treating team, if gastrointestinal bleeding risk was considered higher than average. This variable was not discretely recorded.

Patient demographic, clinical and perioperative data were collected, including the following: age, gender, height, weight, body mass index (BMI), American Society of Anesthesiologists Physical Status (ASA PS) classification, preoperative hemoglobin and hematocrit, estimated intraoperative blood loss, operative time, anesthesia type, length of hospital stay (LOS), perioperative (within 30 days) transfusion or readmission for symptomatic anemia, venous thromboembolic event (VTE) prophylaxis utilized, and discharge disposition.

All statistical analyses were performed using standard software (Microsoft Excel, vers 16.36, 2020). Continuous variables were described using means and standard deviations. Categorical variables were summarized using frequencies and percentages.

\section{Results}

During the study period, 390 consecutive elective primary AA THA were performed by a single surgeon and included in the data analysis. Patient age, gender, height, weight, BMI, and ASA class are reported in Table 2. A wide range of patient age, BMI and ASA score is seen, reflecting a heterogenous population of surgical inpatient candidates. This includes some at higher risk for periop-

Table 2. Patient Demographics

\begin{tabular}{|c|c|c|c|c|}
\hline \multicolumn{3}{|c|}{ Number of patients (n) } & \multicolumn{2}{|l|}{390} \\
\hline \multicolumn{3}{|c|}{ Age (mean + SD, years) } & \multicolumn{2}{|c|}{$\begin{array}{l}64.05+10.67 \text { (range } \\
27-94)\end{array}$} \\
\hline \multicolumn{5}{|l|}{ Sex (n [\%]) } \\
\hline \multicolumn{3}{|l|}{ Male } & \multicolumn{2}{|l|}{$171(44 \%)$} \\
\hline \multicolumn{3}{|l|}{ Female } & \multicolumn{2}{|l|}{$219(56 \%)$} \\
\hline \multicolumn{3}{|c|}{ Height (mean + SD, inches) } & \multicolumn{2}{|c|}{$67.09+3.88$} \\
\hline \multicolumn{3}{|c|}{ Weight (mean + SD, kilograms) } & \multicolumn{2}{|c|}{$86.56+19.71$} \\
\hline \multicolumn{3}{|c|}{$\begin{array}{l}\text { BMI (mean + SD, kilograms/meters } \\
\text { [ㄹ] })\end{array}$} & \multicolumn{2}{|c|}{$\begin{array}{l}29.76+5.98 \text { (range } 16 \\
-47)\end{array}$} \\
\hline ASA PS Class & 1 & 2 & 3 & 4 \\
\hline$(\mathrm{n}[\%])$ & $7(1.8)$ & $178(45.6)$ & $196(50.3)$ & $10(2.6)$ \\
\hline
\end{tabular}

$S D$, standard deviation; BMI, body mass index; ASA PS, American Society of Anesthesiologists Physical Status Classification System 
erative complication given the extremes of age, BMI and ASA score. Preoperative and operative data is presented in Table 3.

No patient required a blood transfusion, or readmission for symptomatic anemia, within the first 30 days postoperative. Preoperative $\mathrm{Hg}$ averaged $13.48 \mathrm{~g} / \mathrm{dL}$. The lowest preoperative $\mathrm{Hg}(9.1 \mathrm{~g} / \mathrm{dL})$ was seen in a patient cleared by their surgeon after lung wedge resection. He underwent routine THA, had no postoperative labs checked and did not require treatment of symptomatic hypotension or require transfusion perioperatively.

Blood loss was surgeon-reported in consultation with the anesthesia team. This averaged $263.88 \mathrm{~mL}$. Three patients had blood loss over $500 \mathrm{~mL}$. Two were large, obese and technically challenging males with prolonged operative times. One of these, who recorded the maximum reported blood loss of $1000 \mathrm{~mL}$, was large (58-year-old male with BMI 40.84) and had massive destructive change. Spinal anesthesia was utilized. The prolonged operative time (109 min) and noted slow ooze from bone preparation was felt to be the reason for the excessive blood loss. His preoperative $\mathrm{Hg}$ was 13.3 and he did have postoperative labs checked. His postoperative day one $\mathrm{Hg}$ was 10.9. He remained asymptomatic and did not require treatment, being discharged on postoperative day (POD) \#1 . The second large male was a similar scenario: 45-year-old male, $195.6 \mathrm{~cm}$ and $181.4 \mathrm{~kg}$, BMI 47.43 , GETA, operative time $174 \mathrm{~min}, \mathrm{EBL} 800 \mathrm{cc}$, and DC POD \#1 without issue. The remaining patient with reported EBL over $500 \mathrm{~mL}$ was an elderly female with chronic obstructive pulmonary disease (COPD), ASA 3 and with poor bone quality. Spinal anesthesia was utilized, and EBL was $600 \mathrm{~mL}$. She did not have postoperative labs checked and did not require treatment, with uneventful discharge POD\#1.

Operative time as derived from the nursing record denotes incision until final dressing placed and shows an average of 91.22 minutes with a fairly narrow distribution (standard deviation $+/-14.20$ minutes).

The majority of patients received a spinal anesthetic $(83.3 \%)$. The cases in which spinal anesthesia was not utilized were due to patient request for general endotracheal anesthesia (GETA), failed attempt at adequate spinal block, or relative contraindication to use (high lumbar spinal fusion or timing of chronic anticoagulation cessation). Of note, mean EBL in patients receiving spinal anesthesia was slightly lower at $259.5 \mathrm{~mL}$ (standard deviation +/- 93.1
$\mathrm{mL}$ ) versus $286.2 \mathrm{~mL}$ with GETA (standard deviation +/103.0). The majority of patients also received Aspirin for VTE prophylaxis (96.7\%), and were discharged on POD \#1 (93.1\%) to home (99\%). Most patients who received an alternative to aspirin for VTE prophylaxis were resuming a chronic anticoagulant.

\section{Discussion}

Excessive blood loss and transfusion are known risks of THA. Reported rates of transfusion in more recent, large studies range from $11.8-22.2 \%[\underline{4}, \underline{6}, \underline{10}, \underline{12}, \underline{15}]$. There is some evidence that the overall rate of transfusion is actually increasing $[4,6]$. These large studies do not qualify transfusion risk based on the approach utilized for THA.

The anterior approach for THA has been associated with increased operative time, complications, and blood loss relative to other approaches [21- $\underline{30]}$. One would suspect an increased transfusion rate associated with the approach given this increased reported blood loss, but no data exists which demonstrates this clearly. Multiple series report blood loss associated with AA THA $[\underline{21}-\underline{28}, \underline{31-}$ 34]. Only three studies were identified which report transfusion data $[\underline{24}, \underline{34}, \underline{35}]$. Alecci et al compared results of 
THA performed by the AA ( $\mathrm{n}=221)$ versus a lateral approach $(n=198)$. They reported a $19.5 \%$ rate of transfusion in the AA group, but this included patients who received either allogenic or autologous blood [35]. Woolson et al [24] noted a high complication and transfusion rate in the learning curve with the AA for five low volume surgeons. They reported a high mean blood loss $(858 \mathrm{~mL})$. They noted an average total transfusion of 2.4 units per patient with most receiving pre-donated blood (most received 1-3 units, average of 1.7 units). The overall transfusion rate is high but not reported, and confounded by autologous predonation [24].

Toy et al [34] present a modern study of 145 AA THA performed in an outpatient surgery center. They report a transfusion rate of $0.7 \%(1 / 145)$. They also note one patient with acute blood loss anemia that required overnight monitoring, but who did not require transfusion. This data is important in that it notes a low transfusion rate with modern AA THA at an outpatient center with a surgeon outside of the procedural learning curve. It adds to growing evidence that the procedure can be performed safely in this setting. The transfusion data in the study, however, is pertinent to the study population. It may not be translatable to the higher risk inpatient setting, as their patients were selected as acceptable candidates for outpatient THA. Their selection criteria included parameters of preoperative HCT $>30$, age $<70$, and BMI $<35$. Their actual patient data reveal an average age of 55 years, and BMI of 29.7. They used a similar TXA protocol as the present study, as well as an albumin bolus during surgery [34]. Their results are comparable to our low rate of transfusion achievable with modern AA THA, but their selection criteria make the transfusion data less applicable to inpatient scenarios.

Understanding the risk factors for transfusion is important to limiting its occurrence. Studies have shown that in THA, increased transfusion rate has been associated with increased weight, lower preoperative $\mathrm{Hg}$, increasing age, lower BMI, higher ASA score, longer operative time, higher surgical EBL, lower surgeon procedural volume, anesthetic type (neuraxial favored), African American race, Medicaid status, and surgery at smaller, rural and nonacademic centers $[\underline{3}, \underline{4}, \underline{6}, \underline{7}, \underline{11}, \underline{12}, \underline{15}]$. Optimizing any of these modifiable risk factors could help minimize transfusion risk.

Transfusion carries significant risk to the patient, and financial detriment to the healthcare system. Patient risk can range from mild to, rarely, life-threatening, and has been well-documented and previously discussed [1- $\underline{3}]$. The occurrence of transfusion in, specifically, joint arthroplasty has been linked to increased LOS, likelihood of discharge to a SNF, and increased cost $[\underline{1}, \underline{2}, \underline{4}-\underline{6}]$. Reported cost for a unit transfusion of blood ranges from $\$ 700-\$ 1,500$ $[\underline{38}, \underline{39}]$, and the impact of transfusion on overall admission cost at $\$ 1,731$ and $\$ 1,777$ in separate studies [4,20]. With transition to the outpatient setting, and bundled care arrangements attempting to link quality outcomes to cost, it is important to avoid this complication.

Following a simple, modern perioperative pathway we have shown the ability to minimize blood loss and the need for transfusion. The basics of this simple pathway emphasize good surgical technique, optimal anesthetic, and the adjunct use of tranexamic acid (TXA) (Table 1).

Surgical cautery devices have been designed to minimize blood loss, with some advanced bipolar sealer devices purporting a lower drop in Hg after THA [40]. We used a standard bovie electrocautery device. The anatomical sites of bleeding the surgeon has to take the utmost care to coagulate, in order of surgical procedural progression, include: isolation and cautery of the ascending branch and accompanying veins of the lateral circumflex artery; slow and meticulous release of the capsule at its attachment to the proximal femur (especially at the inferior neck where more robust vasculature can be present); upon neck cutting, careful attention to minimize past-pointing with the saw blade, causing posterior vascular injury; upon femoral head removal performed in-situ, levering of the head through manipulation of the corkscrew device medially and distally, thus allowing for visualization of the release and coagulation of the previously unobservable posterior capsular structures (as opposed to avulsion of this tissue from the head); after femoral head removal, attention focused to the posterior capsule where disrupted bleeders from the head removal process may be visualized and coagulated; bone wax placement on more aggressively bleeding cancellous bone at the site of the femoral neck cut; upon acetabular preparation, slow and meticulous foveal tissue removal with gentle traction and coagulation systematically; and overall rapid and efficient progression of all surgical steps. This efficiency is particularly important during acetabular preparation and femoral broaching. If bony bleeding occurs during these steps, it cannot be coagulated, and implantation of trial or definitive devices expeditiously can pressurize the bone to limit bleeding. As noted, occasionally, after the femoral neck cut, finger-pressurized bone wax is utilized to reduce bleeding from the femoral cancellous bone. This is primarily done to aid acetabular visualization, as blood can pool at the inferior acetabulum, but in situations with brisk bleeding from the femoral neck the practice can limit overall blood loss. This bone wax is removed at the beginning of the femoral preparation using a curette. A meticulous surgical technique cannot be overemphasized. 
Neuraxial anesthesia has been shown to limit blood loss in THA $[\underline{3}, \underline{10}, \underline{18}]$. In a study of 5,914 cases of both THA and TKA, Turcotte et al found a transfusion rate of $5.8 \%$ with GETA versus $1.6 \%$ for neuraxial $(\mathrm{p}<.001)$. They note that the proposed physiologic reason for this difference seems to be decreased arterial and venous blood pressure, as well as decreased peripheral blood pressure at the actual site of the surgical wound [18]. The use of TXA has also become standard of care for THA, with the American Association of Hip and Knee Surgeons (AAHKS) publishing endorsed, evidence-based, clinical practice guidelines for TXA use in total joint arthroplasty in 2019 [41].

This study is not without limitations. It is a retrospective chart review of electronic medical records, with the inherent limitations associated with such a review process. We recognize too that our EBL data is limited by the selfreported nature of this value. The value was surgeon selfreported with anesthesia team input, but we recognize that these values are never extremely accurate. In addition, it has been recognized that calculation of blood loss through preoperative and postoperative lab values provides a more precise way to account for both operative as well as "hidden" blood loss. Hidden blood loss is noted to account for $26-60 \%$ of total blood loss in hip and knee arthroplasty [3]. We did not routinely check postoperative labs, this not only prevented us from calculating blood loss, it also could have meant that patients with anemia were missed clinically. Nonetheless, we feel that this practice is consistent with most modern THA scenarios, and relevant to increasingly short LOS and cost containment measures (minimizing unnecessary lab draws). Given the overall case number, transfusion data, and lack of readmission for symptomatic anemia, we feel our protocol is supported.

\section{Conclusion}

Transfusion is a known risk in total hip arthroplasty. It has been associated with a multitude of medical complications, increased cost to the healthcare system, and logistical concerns in the outpatient setting. Little data exists regarding the transfusion rate in anterior approach total hip arthroplasty, although many reports suggest increased blood loss relative to other approaches. Our data supports the simple, modern protocol we present as capable of limiting blood loss and transfusion risk in anterior approach total hip arthroplasty.

\section{References}

1. Bolz, N., Zarling, B. \& Markel, D. Long-Term Sustainability of a Quality Initiative Program on Transfusion Rates in Total Joint Arthroplasty: A Follow-up Study. J. Arthroplasty 35, 340-6 (2020). https://doi.org/10.1016/j.arth.2019.08.056

2. Lindman, I. \& Carlsson, L. Extremely Low Transfusion Rates: Contemporary Primary Total Hip and Knee Arthroplasties. J. Arthroplasty 33, 51-4 (2018). https:// doi.org/ 10.1016/j.arth.2017.07.034

3. Newman, J. et al. Quantifying Blood Loss and Transfusion Risk After Primary vs Conversion Total Hip Arthroplasty. J. Arthroplasty 32, 1902-1909 (2017). https:// doi.org/ 10.1016/j.arth.2017.01.038.

4. Saleh, A. et al. Allogenic Blood Transfusion Following Total Hip Arthroplasty: Results from the Nationwide Inpatient Sample, 2000 to 2009. J. Bone Jt. Surg. 96, e155 (2014). https://doi.org/10.2106/JBJS.M.00825.

5. Dunne, J., Malone, D., Tracy, J., Gannon, C. \& Napolitano, L. Perioperative Anemia: An Independent Risk Factor for Infection, Mortality, and Resource Utilization in Surgery. J. Surg. Res. 102, 237-44 (2002). https://doi.org/10.1006/ jsre.2001.6330

6. Browne, J., Adib, F., Brown, T. \& Novicoff, W. Transfusion Rates Are Increasing Following Total Hip Arthroplasty: Risk Factors and Outcomes. J. Arthroplasty 28, 34-7 (2013). https://doi.org/10.1016/j.arth.2013.03.035.

7. Salido, J., Marin, L., Gomez, L., Zorrilla, P. \& Martinez, C. Preoperative Hemoglobin Levels and the Need for Transfusion After Prosthetic Hip and Knee Surgery: Analysis of Predictive Factors. J. Bone Jt. Surg. 84, 216-20 (2002). https:// doi.org/10.2106/00004623-200202000-00008.

8. Weber, E. et al. Perioperative Blood Transfusions and Delayed Woundd Healing After Hip Replacement Surgery: Effects on Duration of Hospitalization. Anesth. Analg. 100, 1416-21 (2005). https://doi.org/10.1213/01. ANE.0000150610.44631.9D.

9. Wolf, B., Lu, X., Li, Y., Callaghan, J. \& Cram, P. Adverse Outcomes in Hip Arthroplasty: Long-Term Trends. J. Bone Jt. Surg. 94, e103 (2012). https://doi. org/10.2106/JBJS.K.00011.

10. Park, J. et al. Predictors of Perioperative Blood Loss in Total Joint Arthroplasty. J. Bone Jt. Surg. 95, 1777-83 (2013). https://doi.org/10.2106/JBJS.L.01335.

11. Frisch, N. et al. Predictors and Complications of Blood Transfusion in Total Hip and Knee Arthroplasty. J. Arthroplasty 29, 189-92 (2014) . https://doi. org/10.1016/j.arth.2014.03.048

12. Hart, A. et al. Blood Transfusion in Primary Total Hip and Knee Arthroplasty. Incidence, Risk Factors, and Thirty-Day Complication Rates. J. Bone Jt. Surg. 93, 1945-51 (2014). https://doi.org/10.2106/JBJS.N.00077.

13. Yoshihara, H. \& Yoneoka, D. National Trends in the Utilization of Blood Transfusions in Total Hip and Knee Arthroplasty. J. Arthroplasty 29, 1932-7 (2014). https://doi.org/10.1016/j.arth.2014.04.029.

14. Carling, M., Jeppsson, A., Eriksson, B. \& Brisby, H. Transfusion and Blood Loss in Total Hip and Knee Arthroplasty: A Prospective Observational Study. J. Orthop. Surg. 10, 48 (2015). https://doi.org/10.1186/s13018-015-0188-6.

15. Menendez, M. et al. Variation in Use of Blood Transfusion in Primary Total Hip and Knee Arthroplasty. J. Arthroplasty 31, 2757-63 (2016). https://doi org/10.1016/j.arth.2016.05.022.

16. Ondeck, N. et al. Predicting Adverse Outcomes After Total Hip Arthroplasty: A Comparison of Demographics, the American Society of Anesthesiologists class, the Modified Charlson Comorbidity Index, and the Modified Frailty Index. J. Am. Acad. Orthop. Surg. 26, 735-43 (2018). https://doi.org/10.5435/JAAOSD-17-00009.

17. Greenky, M., Wang, W., Ponzio, D. \& Courtney, P. Total Hip Arthroplasty and the Medicare Inpatient-Only List: An Analysis of Complications in Medicare-Aged Patients Undergoing Outpatient Surgery. J. Arthroplasty 34, 1250-54 (2019). https://doi.org/10.1016/j.arth.2019.02.031

18. Turcotte, J., Stone, A., Gilmor, R., Gormica, J. \& King, P. The Effect Neuraxial Anesthesia on Postoperative Outcomes in Total Joint Arthroplasty with Rapid Recovery Protocols. J. Arthroplasty 35, 950-4 (2020). https://doi.org/10.1016/j. arth.2019.11.037

19. Nelson, S. et al. Is Outpatient Total Hip Arthroplasty Safe? J. Arthroplasty 32, 1439-42 (2017). https://doi.org/10.1016/j.arth.2016.11.053.

20. Klika, A. et al. Primary Total Knee Arthroplasty Allogenic Transfusion Trends, Length of Stay, and Complications: Nationwide Inpatient Sample 2000-2009. J. Arthroplasty 29, 2070-7 (2014). https://doi.org/10.1016/j.arth.2014.06.018.

21. Anterior Total Hip Arthroplasty Collaborative Investigators et al. Outcomes following the single-incision anterior approach to total hip arthroplasty: a multicenter observational study. Orthop. Clin. N. Am. 40, 329-42 (2009). https://doi. org/10.1016/j.ocl.2009.03.001. 
22. D'Arrigo, C., Speranza, A., Monaco, E., Carcangiu, A. \& Ferretti, A. Learning curve in tissue sparing total hip replacement: comparison between different approaches. J. Orthop. Traumatol. 10, 47-54 (2009). https://doi.org/10.1007/s10195008-0043-1.

23. Nakata, K., Nishikawa, M., Yamamoto, K., Hirota, S. \& Yoshikawa, H. A Clinical Comparative Study on the Direct Anterior with Mini-Posterior Approach: Two Consecutive Series. J. Arthroplasty 24, 698-704 (2009). https://doi.org/10.1016/j. arth.2008.04.012.

24. Woolson, S., Pouliot, M. \& Huddleston, J. Primary Total Hip Arthroplasty Using an Anterior Approach and a Fracture Table: Short-term Results from a Community Hospital. J. Arthroplasty 24, 999-1005 (2009). https://doi.org/10.1016/j. arth.2009.04.001.

25. Hallert, O., Li, Y. \& Lindgren, U. The direct anterior approach: initial experience of a minimally invasive technique for total hip arthroplasty. J. Orthop. Surg. 7, 17 (2012). https://doi.org/10.1186/1749-799X-7-17.

26. Spaans, A., van den Hout, J. \& Bolder, S. High Complication Rate in the Early experience of Minimally Invasive Total Hip Arthroplasty by the Direct Anterior Approach. Acta Orthop. 83, 342-6 (2012). https://doi.org/10.3109/17453674.20 12.711701 .

27. Zawadsky, M., Paulus, M., Murray, P. \& Johansen, M. Early Outcome Comparison Between the Direct Anterior Approach and the Mini-Incision Posterior Approach for Primary Total Hip Arthroplasty: 150 Consecutive Cases. J. Arthroplasty 29, 1256-60 (2014). https://doi.org/10.1016/j.arth.2013.11.013.

28. Jewett, B. \& Collis, D. High Complication Rate with Anterior Total Hip Arthroplasties on a Fracture Table. Clin. Orthop. 469, 503-7 (2011). https://doi. org/10.1007/s11999-010-1568-1.

29. Christensen, C., Karthikeyan, T. \& Jacobs, C. Greater Prevalence of Wound Complications Requiring Reoperation with Direct Anterior Approach Total Hip Arthroplasty. J. Arthroplasty 29, 1839-1841 (2014). https://doi.org/10.1007/s11999-010$1568-1$.

30. Lee, G. \& Marconi, D. Complications Following Direct Anterior Hip Procedures: Costs to Both Patients and Surgeons. J. Arthroplasty 30, 98-101 (2015). https:// doi.org/10.1016/j.arth.2015.03.043.

31. Barrett, W., Turner, S. \& Leopold, J. Prospective Randomized Study of Direct Anterior vs Postero-Lateral Approach for Total Hip Arthroplasty. J. Arthroplasty 28, 1634-8 (2013). https://doi.org/10.1016/j.arth.2013.01.034.

32. Martin, C., Pugely, A., Gao, Y. \& Clark, C. A Comparison of Hospital Length of Stay and Short-term Morbidity Between the Anterior and the Posterior Approaches to Total Hip Arthroplasty. J. Arthroplasty 28, 849-54 (2013). https://doi. org/10.1016/j.arth.2012.10.029.

33. Barnett, S. et al. Is the Anterior Approach Safe? Early Complication Rate Associated with 5090 Consecutive Primary Total Hip Arthroplasty Procedures Performed Using the Anterior Approach. J. Arthroplasty 31, 2291-4 (2016). https:// doi.org/10.1016/j.arth.2015.07.008.

34. Toy, P., Fournier, M., Throckmorton, T. \& Mihalko, W. Low Rates of Adverse Events Following Ambulatory Outpatient Total Hip Arthroplasty at a Free-Standing Surgery Center. J. Arthroplasty 33, 46-50 (2018). https://doi.org/10.1016/j. arth.2017.08.026.

35. Alecci, V., Valente, M., Minerva, M., Pellegrino, C. \& Sabbadini, D. Comparison of primary total hip replacements performed with a direct anterior appproach versus the standard lateral approach: perioperative findings. J. Orthop. Traumatol. 12, 123-9 (2011). https://doi.org/10.1007/s10195-011-0144-0.

36. Matta, J., Shahrdar, C. \& Ferguson, T. Single-incision Anterior Approach for Toal Hp Arthroplasty on an Orthopaedic Table. Clin. Orthop. 411, 115-24 (2005). https://doi.org/10.1097/01.blo.0000194309.70518.cb.

37. Carson, J. et al. Red Blood Cell Transfusion: A Clinical Practice Guideline from the AABB*. Ann. Intern. Med. 157, 49-58 (2012). https://doi.org/10.7326/00034819-157-1-201206190-00429.

38. Tuttle, J. et al. Cost Benefit Analysis of Topical Tranexamic Acid in Primary Total Hip and Knee Arthroplasty. J. Arthroplasty 29, 1512-5 (2014). https://doi. org/10.1016/j.arth.2014.01.031.

39. Blumberg, N., Kirkley, S. \& Heal, J. A Cost Analysis and Allogenic Transfusions in Hip-replacement Surgery. Am. J. Surg. 171, 324-30 (1996). https://doi. org/10.1016/S0002-9610(97)89635-3.

40. Marulanda, G., Ulrich, S., Seyler, T., Delanois, R. \& Mont, M. Reductions in Blood Loss with Bipolar Sealer in Total Hip Arthroplasty. Expert Rev. Med. Devices 5, 125-31 (2008). https://doi.org/10.1586/17434440.5.2.125.

41. Fillingham, Y. et al. Tranexamic Acid in Total Joint Arthroplasty: The Endorsed Clinical Practice Guides of the American Association of Hip and Knee Surgeons, American Society of Regional Anesthesia and Pain Medicine, American Academy of Orthopaedic Surgeons, The Hip Society, and the Knee Society. Reg. Anesth. Pain Med. 44, 7-11 (2019). https://doi.org/10.1016/j.arth.2018.08.002.
SUBMISSION HISTORY

Submitted: August 7, 2020

Reviewed: October 10, 2020

Revised October 19, 2020

Accepted: December 18, 2020

Published: January 30, 2021

\section{AUTHOR AFFILIATIONS}

1 Dr Joseph Gondusky MD

Hip and Knee Arthroplasty Surgeon

Jordan-Young Institute, Virginia Beach, VA

2 Dr Benjamin Campbell MD

Thomas Jefferson University, Philidelphia, PA

3 Christian Coulson

Eastern Virginia Medical School, Norfolk, VA

(Direct inquires to Joseph Gondusky, josephgondusky@gmail.com)

AUTHOR DISCLOSURES

- The authors declare that there is no conflict of interest in connection with this submitted article.

\section{COPYRIGHT \& OPEN ACCESS}

(C) 2021 Gondusky, Campbell, Coulson. All rights reserved. Authors retain copyright and grant the journal right of first publication with the work. Reconstructive Review is an open access publication and follows the Creative Commons Attribution-NonCommercial CC BY-NC. This license allows anyone to download works, build upon the material, OPEN $O$ ACCEss and share them with others for non-commercial purposes as long as they credit the senior author, Reconstructive Review, and the Joint Implant Surgery \& Research Foundation (JISRF). An example credit would be: "Courtesy of (senior author's name), Reconstructive Review, JISRF, Chagrin Falls, Ohio". 